\title{
Heat shock protein 60: an endogenous inducer of dopaminergic cell death in Parkinson disease
}

Carmen Noelker ${ }^{1,2}$, Lydie Morel ${ }^{1}$, Anke Osterloh ${ }^{3}$, Daniel Alvarez-Fischer ${ }^{1,2,4,5}$, Thomas Lescot ${ }^{1}$, Minka Breloer $^{3}$, Maike Gold ${ }^{2}$, Wolfgang H Oertel ${ }^{2}$, Carmen Henze ${ }^{1}$, Patrick P Michel ${ }^{1}$, Richard C Dodel ${ }^{2}$, Lixia Lu' ${ }^{1}$ Etienne C Hirsch?', Stéphane Hunot ${ }^{1 *}$ and Andreas Hartmann ${ }^{1 *}$

\begin{abstract}
Background: Increasing evidence suggests that inflammation associated with microglial cell activation in the substantia nigra (SN) of patients with Parkinson disease (PD) is not only a consequence of neuronal degeneration, but may actively sustain dopaminergic (DA) cell loss over time. We aimed to study whether the intracellular chaperone heat shock protein 60 (Hsp60) could serve as a signal of CNS injury for activation of microglial cells.

Methods: Hsp60 mRNA expression in the mesencephalon and the striatum of C57/BL6 mice treated with MPTP (1-methyl-4-phenyl-1,2,3,6-tetrahydropyridine) and the Hsp60/TH mRNA ratios in the SN of PD patients and aged-matched subjects were measured. To further investigate a possible link between the neuronal Hsp60 response and PD-related cellular stress, Hsp60 immunoblot analysis and quantification in cell lysates from SH-SY5Y after treatment with $100 \mu \mathrm{M} \mathrm{MPP}^{+}$(1-methyl-4-phenylpyridinium) at different time points (6, 12, 24 and 48 hours) compared to control cells were performed. Additional MTT and LDH assay were used. We next addressed the question as to whether Hsp60 influences the survival of $\mathrm{TH}^{+}$neurons in mesencephalic neuron-glia cultures treated either with $\mathrm{MPP}^{+}(1 \mu \mathrm{M})$, hHsp60 $(10 \mu \mathrm{g} / \mathrm{ml})$ or a combination of both. Finally, we measured IL-1 $\beta$, IL-6, TNF-a and NO-release by ELISA in primary microglial cell cultures following treatment with different hHsp60 preparations. Control cultures were exposed to LPS.

Results: In the mesencephalon and striatum of mice treated with MPTP and also in the SN of PD patients, we found that Hsp60 mRNA was up-regulated. MPP ${ }^{+}$, the active metabolite of MPTP, also caused an increased expression and release of Hsp60 in the human dopaminergic cell line SH-SY5Y. Interestingly, in addition to being toxic to DA neurons in primary mesencephalic cultures, exogenous Hsp60 aggravated the effects of $\mathrm{MPP}^{+}$. Yet, although we demonstrated that Hsp60 specifically binds to microglial cells, it failed to stimulate the production of pro-inflammatory cytokines or NO by these cells.
\end{abstract}

Conclusions: Overall, our data suggest that Hsp60 is likely to participate in DA cell death in PD but via a mechanism unrelated to cytokine release.

Keywords: PD, Neuroinflammation, Hsp60, Neurodegeneration, Microglia, Innate immunity

\footnotetext{
* Correspondence: stephane.hunot@upmc.fr; andreas.hartmann@psl.aphp.fr

'CR-ICM, INSERM UMR_S1127, Université Pierre et Marie Curie Paris 06

UMR_S1127, CNRS UMR 7225, Groupe Hospitalier Pitié-Salpêtrière, 75013

Paris, France

Full list of author information is available at the end of the article
} 


\section{Background}

Parkinson disease (PD) is a common neurodegenerative disorder clinically characterized by akinesia, rigidity and rest tremor [1]. The cardinal neuroanatomical feature of $\mathrm{PD}$ is a massive and preferential loss of dopaminergic (DA) neurons in the substantia nigra pars compacta $(\mathrm{SNpc})$ of patients, resulting in a drastic decline in striatal dopamine concentrations.

Another histopathologic hallmark of PD is microglial activation in the SNpc [2,3]. Activated microglia are believed to contribute to the neurodegenerative process through the release of pro-inflammatory and/or cytotoxic factors such as IL-1 $\beta$, TNF- $\alpha$, nitric oxide (NO) and reactive oxygen intermediates [2]. Moreover, several observations suggest that an ongoing stimulus could lead to disease progression long after the initial toxic insult [4-6], thereby amplifying and sustaining neuroinflammation and, ultimately, leading to destruction of nigral DA neurons. These processes are generally considered to be a non-specific consequence of neuronal death. But the possibility that suffering/damaged DA neurons might also participate in the activation of microglia and in sustaining neuroinflammation deserves consideration.

In light of our knowledge about the innate immune system, it is highly plausible to assume that Toll-like receptors (TLRs) are major mediators in glial cells triggering the release of cytokines that ultimately kill DA neurons in the SNpc. Accordingly, as first demonstration in the central nervous system (CNS), the only cellular population that stained positive for Toll-like receptor 4 (TLR4) in the brain parenchyma of adult rats were microglia [7].

Traditionally, it has been considered that the dangerassociated molecules sensed by TLRs are highly conserved, so called pathogen-associated molecular patterns (PAMPs), which are expressed by bacteria, viruses, or other pathogens but are not present in mammalian cells [8]. For example, bacterial lipopeptides (BLPs), lipopolysaccharide (LPS), and flagellins are recognized by TLR2, TLR4, and TLR5, respectively. A number of reports have emerged to suggest that diverse molecules of host-cell origin may also serve as endogenous ligands of TLR2 or TLR4 [9]. To date, there have been at least 23 reports of distinct endogenous ligands of TLR2 or TLR4, representing molecules of diverse source and structure, ranging from those associated with cell damage and major extracellular matrix (ECM) turnover to inflammatory mediators and oxidatively modified lipids [10].

Recently, we have demonstrated that TLR4-deficient mice are less vulnerable to MPTP (1-methyl-4-phenyl1,2,3,6-tetrahydropyridine) intoxication than wild-type mice and display a decreased number of $\mathrm{Iba}^{+}$and MHC $\mathrm{II}^{+}$activated microglial cells after MPTP application, suggesting that the TLR4 pathway is involved in experimental PD. However, the TLR4 ligand responsible for this activation remains elusive [7].

Neuronal activators of the TLR4-dependent pathway in microglia could be heat shock proteins (Hsps), a group of highly conserved proteins that are constitutively expressed in most cells under physiological conditions. They are commonly induced by the presence of denatured proteins and contribute to the restoration of the tertiary structure and enzymatic activity of these proteins [11]. Upon cellular stress, Hsps are up-regulated and released from suffering/damaged cells $[12,13]$. Beside the assumption that the Hsp response to cell injury plays a beneficial role in cell survival, recent evidence suggests that Hsps can also exert immunomodulatory functions [14]. Hsp60 - which is typically located in mitochondria, a primary subcellular pathogenic locus in PD [15] - was found to be aberrantly expressed on the cell surface of neuronal cells in response to stress $[16,17]$. Interestingly, Hsp60 binds to microglia and the microglial receptor for Hsp60 has been suggested to be TLR4 [18]. In addition, a recent study demonstrated that Hsp60 serves as an endogenous signal of injury in the CNS by activating microglia via a TLR4- and myeloid differentiation factor 88 (MyD88)-dependent pathway [19]. This mechanism may account for the triggering of inflammatory responses in the vicinity of suffering/damaged DA neurons and engage a neuronal-glial crosstalk. We therefore investigated the role of Hsp60 in this scenario which may be released or expressed at the cell surface of suffering/damaged DA neurons and contribute to their demise.

\section{Material and methods}

\section{Animals and MPTP treatment}

Adult (eight to ten weeks) male C57/BL6 mice (Janvier Breeding Centre, France) were housed, handled, and cared for in accordance with the Guide for the Care and Use of Laboratory Animals (NCR (National Research Council) 1996) and the European Union Council Directive 86/609/ EEC, and the experimental protocols were carried out in compliance with institutional ethical committee guidelines for animal research. For all studies, mice were maintained on a 12:12 hour light/dark cycle with lights on at $6.30 \mathrm{am}$. The room temperature was kept at $23^{\circ} \mathrm{C}$, with free access to standard diet (LASQCdiet ${ }^{\oplus} \operatorname{Rod} 16-\mathrm{R}$, LASvendi) and tap water. Mice ( $n=8$ to 10 per group) were treated using the acute MPTP paradigm $(4 \times 20 \mathrm{mg} / \mathrm{kg} \mathrm{MPTP}-\mathrm{HCl}$ (Sigma-Aldrich, Lyon, France) every 2 hours) [20,21] and animals were sacrificed after 12, 24, 48, 96 or 168 hours after the last injection. The brain was excised and the specific brain regions dissected. The corresponding controls were collected variously across the days.

\section{Hsp60 mRNA-PCR in the striatum and SN of mice}

Striatum and SN of the mice were dissected. Tissues were then placed into Trizol reagent (Invitrogen, Cergy 
Pontoise Cedex, France), homogenized, and total RNA was prepared according to the manufacturer's instructions. First-strand cDNA was synthesized from $1 \mu \mathrm{g}$ of total RNA using the ThermoScript RT-PCR system (Invitrogen, Cergy Pontoise Cedex, France). To access expression levels specific designed intron-spanning primers for Hsp60 (fwd $=5^{\prime}$-CAC AGT GAA GGA TGG AAA AAC CCT-3' and rev $=5^{\prime}-\mathrm{TCT}$ TTG GTG ACA ATG ACC TCC C-3') and tubulin (fwd $=5^{\prime}-$ TGT CCA TGA AGG AGG TGG ATG AG-3' and rev = 5' ATG TTG CTC TCA GCC TCG GTG AAC-3') were used. The annealing temperature was $64^{\circ} \mathrm{C}$ and the number of PCR cycles was chosen to stop the reaction in the linear phase of amplification ( 25 cycles). The amplification of Hsp60 and tubulin was done in the same reaction for each sample. After gel electrophoresis, digital images were analyzed with NIH ImageJ software for quantification.

\section{Hsp60 qRT-PCR of human SN}

The Hsp60 and TH primers for quantitative PCR were designed by primer express 3.0 (Applied Biosystems, Darmstadt, Germany) and their specificity was confirmed by blastn analysis. Their sequences were as followed: Hsp60_fwd: 5'-GCC GCC CCG CAG AA-3', Hsp60_rev: 5'-CCT GGA CAC CGG TCT CAT CT-3', TH_fwd: 5' GCA CCT TCG CGC AGT TCT-3', TH_rev: 5'-ACA GCG TGG ACA GCT TCT CA-3'. Mesencephalon ss PD patients and age-matched control subjects $(n=5$ per group) were provided by the INSERM UMR_S1127 brain bank. The SN was dissected from frozen slides. Total RNA was extracted with Trizol (Invitrogen, Cergy Pontoise Cedex, France) and the quantity and quality were determined by Nanodrop and Agilent, respectively. One microgram of total RNA was reverse-transcribed with Superscript III RT-kit (Invitrogen, Cergy Pontoise Cedex, France) according to the manufacturer's instructions. qPCR was performed with SYBR GreenER TM qPCR SuperMix kit (Invitrogen, Cergy Pontoise Cedex, France) in ABI 7500 real time thermal cycler (Applied Biosystems, Darmstadt, Germany). The relative mRNA expression levels were normalized by the geometric mean of two housekeeping genes (GAPDH and HPRT) with qBaseplus software (Primerdesign, Southampton, UK).

\section{SH-SY5Y cell cultures}

SH-SY5Y (p3-p7) cells [22] were maintained in DMEM (Gibco, Saint Aubin, France) supplemented with 15\% FCS, $1 \mathrm{mM}$ L-glutamine, $100 \mathrm{U}$ of penicillin/ml, and $100 \mu \mathrm{g}$ of streptomycin sulfate $/ \mathrm{ml}$ and equilibrated with $5 \% \mathrm{CO}_{2}$ $95 \%$ air at $37^{\circ} \mathrm{C}$. At different time points, the cells were treated with $100 \mu \mathrm{M} \mathrm{MPP}{ }^{+}$(1-methyl-4-phenylpyridinium) [23] purchased from Sigma-Aldrich (Lyon, France).

\section{Primary mesencephalic cell cultures}

Mesencephalic cultures were prepared from the ventral midbrain of Swiss mouse embryos at gestational age 13.5 days (Janvier Breeding Center, France). The dissected tissue pieces were processed according to previously described protocols $[24,25]$. Briefly, after mechanical dissociation in modified L15 medium [26] with no enzymatic treatment, the cells in suspension were plated at a density of 1.5 to $2.0 \times 10^{5}$ cells $/ \mathrm{cm}^{2}$ in polyethylenimine (PEI, Sigma-Aldrich, Lyon, France; $1 \mathrm{mg} / \mathrm{mL}$ ) precoated culture plates (24 well-plates). The cells were then allowed to mature and differentiate in N5 culture medium [25,27], supplemented with $5 \%$ horse serum and $0.5 \%$ fetal calf serum (FCS, endotoxin level of the used FCS $<10 \mathrm{EU} / \mathrm{ml}$ ) except for the first three days in vitro (DIV), when the concentration of FCS was raised to $2.5 \%$ to favor cell attachment and initial development. Mesencephalic DA neurons degenerate spontaneously and progressively when maintained in N5 culture medium supplemented with serum proteins, whereas other types of neurons survive. The death of these neurons occurs spontaneously through a mechanism that is dependent on glial cells [24,28]. It could be shown previously that depolarization by elevation of extracellular $\mathrm{K}^{+}\left(\left[\mathrm{K}^{+}\right] 30 \mathrm{mM}\right)$ was efficient in preventing DA cell demise. Note that $\mathrm{K}^{+}$-induced depolarization was performed in the presence of $1 \mu \mathrm{M}$ MK-801 to prevent secondary excitotoxic stress. In some experiments, Ara-C $(2 \mu \mathrm{M})$ was added to the medium at DIV 1 to 2 after plating to inhibit proliferation of non-neuronal cells (astrocytes, microglia) as well in the presence of $1 \mu \mathrm{M}$ MK-801 to prevent secondary excitotoxic stress [28].

Mesencephalic cultures were treated either with $1 \mu \mathrm{M}$ $\mathrm{MPP}^{+}$at DIV 4 and DIV 5 as previously described [29], $10 \mu \mathrm{g} / \mathrm{ml}$ low-endotoxin recombinant human Hsp60 dissolved in PBS (hHsp60; Loke Diagnostics ApS, Risskov, Denmark) at DIV 2 to DIV 5 or in combination of both. The hHsp60 contained $<2$ endotoxic units of LPS/mg of protein as determined by limulus amoebocyte lysate assay (BioWhittaker, Cologne, Germany). Since hHsp60 has a much weaker effect than $\mathrm{MPP}^{+}$on cell death, we decide to prolonged treatment of the cell cultures compared to $\mathrm{MPP}^{+}$treatment. At DIV 5, medium including $\mathrm{MPP}^{+}$and hHsp60 was removed and cultures were left to recover until DIV 10 in control medium. Trypsin proteolysis (Gibco, Saint Aubin, France) or pre-treatment by boiling of hHsp60 were used to control for Hsp60-mediated effects, as well as additional treatment with polymyxin B $(10 \mu \mathrm{g} / \mathrm{ml}$, PMBS, Sigma-Aldrich, Lyon, France) as a specific LPS inhibitor to control for LPS effects.

\section{Highly enriched microglial cultures of mice ventral mesencephalon}

Almost pure microglial cultures were obtained using a technique of high-yield isolation of microglia by mild 
trypsinization [30]. Briefly, neuronal/glial mesencephalic cultures were prepared as described previously except for the culture medium, which was DMEM/F12 nutrient mixture (DMEM/F12; Invitrogen, Cergy Pontoise, France) supplemented with 10\% FCS (endotoxin level of the used FCS $<10$ EU/ml). After DIV 14, the cultures were washed for 1 minute with DMEM/F12 to eliminate serum and then incubated with a trypsin/EDTA solution (0.25\% trypsin, $1 \mathrm{~mm}$ EDTA in HBSS; Invitrogen, Cergy Pontoise, France) diluted 1:4 in DMEM/F12 for half to one hour at $37^{\circ} \mathrm{C}$ until the upper layer (mainly constituted by neurons/ astrocytes) was detached. The medium containing the layer of detached cells was aspirated and the highly enriched microglial cell population ( $98 \%$ of pure microglial cells) that remained attached to the bottom of the well was exposed to $500 \mu \mathrm{l}$ of DMEM/F12 with $10 \%$ FCS to allow trypsin inactivation. The cells were treated with lowendotoxin recombinant human Hsp60 (hHsp60) $10 \mu \mathrm{g} / \mathrm{ml}$ and contained $<2$ endotoxic units of LPS/mg of protein as determined by limulus amoebocyte lysate assay (BioWhittaker, Cologne, Germany) or LPS $0.1 \mu \mathrm{g} / \mathrm{ml}$ (Sigma-Aldrich, Lyon, France, strain 055:B5), both dissolved in PBS.

\section{MTT and LDH assays}

MTT (3-[4,5-dimethylthiazol-2-yl]-2,5-diphenyl-tetrazolium bromide, Sigma-Aldrich, Lyon, France) was added to the cells at a final concentration of $0.25 \mathrm{mg} / \mathrm{ml}$ and incubated for 1 hour [31-33]. Thereafter, the cells were lysed and quantified using a microplate reader (MultiskanReader, ThermoLabsystems, Egelsbach, Germany) at a wavelength of $570 \mathrm{~nm}$. Cell death and cell lysis was based on lactate dehydrogenase (LDH) activity released into the supernatant. LDH activity was measured using the CytoTox $96^{\circ}$ Non-Radioactive Cytotoxicity Assay (Promega, Lyon, France) according to the manufacturer's protocol.

\section{Western blots}

For Western blotting, cellular protein was isolated using the lysis buffer M-PER Mammalian Protein Extraction Reagent according to the manual (Pierce, Perbio, Brebières, France). Twenty micrograms total protein/lane was loaded onto a reducing $12 \%$ SDS-PAGE and electroblotted onto nitrocellulose membranes. After blotting, the internal protein loading controls were prepared according to the manual of MEM Code, Pierce (Perbio, Brebières, France). Afterwards, the membranes were blocked and incubated for 24 hours at $4{ }^{\circ} \mathrm{C}$ with a monoclonal mouse anti-Hsp60 antibody $(0.5 \mu \mathrm{g} / \mathrm{ml}$; R\&D Systems, Wiesbaden, Germany) and for the loading control with an anti-beta-actin polyclonal rabbit antibody (1:2,000, Sigma-Aldrich, Lyon, France), respectively. For detection, the nitrocellulose membranes were washed and incubated with a HRP- conjugated secondary antibody (Jackson ImmunoResearch, West Grove, PA, USA; goat-anti-mouse or goatanti-rabbit both 1:50,000). After AB-incubation membranes were incubated in Super Signal Ultra substrate working solution (Perbio, Brebières, France) and exposed to an autoradiographic film (Mat Plus DG Film, Kodak, MaisonsAlfort, France). Afterwards, Western blot signals were analyzed by densitometry using the NIH Image software Scion, and the Hsp60/ $\alpha$-actin ratio was calculated.

\section{Immunocytochemical procedures in vitro}

All cell cultures were fixed for 15 minutes at room temperature with formaldehyde (4\% in PBS) and washed three times with PBS. For primary mesencephalic cell cultures, the survival of DA neurons was determined by tyrosine hydroxylase $(\mathrm{TH})$ immunocytochemistry. The cultures exposed first for 24 hours at $4^{\circ} \mathrm{C}$ to a rabbitpolyclonal anti-TH antibody (Pel-Freez Biologicals, Rogers, AR, USA) diluted 1:1,000 in PBS containing 0.2\% Triton $\mathrm{X}-100$, were then incubated for one hour at room temperature with an anti-rabbit IgG Alexa488 conjugate (1:500; Sigma-Aldrich/RBI, Lyon, France). In order to identify the total number of neurons in the cultures, cultures were incubated for 48 hours at $4^{\circ} \mathrm{C}$ with a mouse anti-neuronal Nuclei (NeuN) biotin conjugated monoclonal antibody (Chemicon International, Limburg, Germany) and then incubated with a streptavidin-Cy3-antibody (1:1,000, Jackson ImmunoResearch, West Grove, PA, USA) for 1 hour at room temperature. Mesencephalic cultures contained between 1 and $3 \% \mathrm{TH}^{+}$cells at the time of plating. To quantify the cells, the entire $\mathrm{TH}^{+}$and/or NeuN ${ }^{+}$neurons of the used wells were counted.

In the primary microglial cultures, microglial cells were identified with a rat anti-CD11b Ig (MAC-1; Serotec, Oxfordshire, UK; 1:100 in PBS, 24 hours at $4^{\circ} \mathrm{C}$ ) and revealed using an anti-rabbit IgG Alexa488 conjugate (1:500; Sigma-Aldrich/RBI, Lyon, France). Note that Triton X-100 was not used for the detection of microglial cell surface markers. A polyclonal rabbit beta-tubulin antibody was used (Cell Signaling, Leiden, Netherlands; 1: 200 in PBS containing $0.2 \%$ Triton $\mathrm{X}-100,24$ hours at $4^{\circ} \mathrm{C}$ ) to detect cytoskeletal structures and revealed using an anti-rabbit IgG Alexa488 conjugate (1:500; Sigma-Aldrich/RBI, Lyon, France).

For staining double-stranded DNA, we used PBS containing $10 \mu \mathrm{g} / \mathrm{ml}$ 4'6-diamidino-2-phenylindole (DAPI, Sigma-Aldrich, Lyon, France). Illustrations of microglial cells are presented as inverted images. For the confocal images, a Leica SP1 confocal microscope was used (Leica, Wetzlar, Germany).

\section{Hsp60 binding studies}

For binding studies, labeled or unlabeled hHsp60 and BSA (Sigma-Aldrich, Lyon, France) were used. The labeled 
proteins were conjugated to Alexa 647 using the protein labeling kits from Molecular Probes, Saint Aubin, France, according to the manufacturer's protocol. For binding of hHsp60 to primary microglia $1 \times 106$ cells were incubated on ice with either $10 \mu \mathrm{g} / \mathrm{ml}$ Alexa 647-labeled hHsp60 or BSA. To exclude an unspecific effect of labeled proteins, cells were also treated with unlabeled proteins (hHsp60 or BSA). Cells that obtained unlabeled hHsp60 were treated with $30 \mu \mathrm{l}$ of Cohn II fraction (SigmaAldrich, Lyon, France) and stained with Hsp60-specific antibody (clone LK-1, 1:100 in PBS), TRITC-labeled goat anti-mouse secondary antibody (1:400 in PBS) and DAPI (1:1,000 in PBS). After staining cells were fixed in PBS/1\% paraformaldehyde (PFA), centrifuged onto glass slides, and covered with anti-FADE solution (BiomedDia, Zweibruechen, Germany).

\section{Hsp60 quantification}

The estimation of protein concentration was performed by the Bradford assay and verified by SDS-PAGE followed by silver staining. Western blot for Hsp60 quantification was made with a specific anti-Hsp60 monoclonal antibody (SPA-810, StressGen, San Diego, CA, USA).

\section{FACS staining}

1 to $2 \times 10^{6}$ microglial cells were washed with PBS, detached and incubated with different concentrations of hHsp60 (1 to $50 \mu \mathrm{g} / \mathrm{ml}$ ) on ice for 30 minutes. Cells were washed with PBS and Fc receptors of cells were blocked with $30 \mu \mathrm{l}$ of Cohn II fraction $(10 \mathrm{mg} / \mathrm{ml}$, Sigma-Aldrich, Lyon, France) prior to staining with a specific antibody against Hsp60 (SPA-810; StressGen, San Diego, CA, USA). To avoid an internalization of cell surface-bound Hsp60, the incubation of microglial cells with Hsp60 was done on ice. Cells were then washed and a phycoerythrin-labeled goat-anti mouse IgG (Jackson ImmunoResearch, West Grove, PA, USA) was added.

\section{Cytokine and Hsp60 quantification by ELISA}

The supernatant of SH-SY5Y cell cultures was collected at different time points, centrifuged and immediately frozen at $-80^{\circ} \mathrm{C}$. The detection of Hsp60 was carried out by using a quantitative sandwich immunoassay (Hsp60ELISA-Kit, StressGen, San Diego, CA, USA) according to the manufacturer's protocol. Release of microglial cytokines (IL-1 $\beta$, IL- 6 and TNF- $\alpha$ ) was measured at different time points (1, 2, 4, 6 and 24 hours) after addition of $10 \mu \mathrm{g} / \mathrm{ml} \mathrm{hHsp60.}$

In addition, microglial cells were treated with hHsp60 that was left untreated, boiled, trypsin-inactivated or pre-incubated with either a specific Hsp60-antibody (Clone 4B9; Dianova, Hamburg, Germany) or an isotypic IgG2-antibody (Santa Cruz Biotechnology Inc., Heidelberg, Germany). As a positive control for cytokine release, primary microglial cells were incubated with only LPS $(0.1 \mu \mathrm{g} / \mathrm{ml})$, a prototypical inflammogen known to activate microglia through TLR4 stimulation $[34,35]$.

Cytokine release into the supernatant was measured employing the DuoSet ELISA Development System mouse IL-1 $\beta$, IL- 6 and TNF- $\alpha$ (R\&D Systems, Wiesbaden, Germany). ELISAs were performed according to the manufacturer's protocol.

\section{Measurement of NO}

Accumulated nitrite, a stable oxidation product of $\mathrm{NO}$, was measured using Griess reagents [36]. Fifty microliters of primary microglial cell supernatants were transferred to a 96 -well microtiter plate and $50 \mu \mathrm{l}$ of solution 1 (1\% sulfanilamide in $5 \%$ phosphoric acid) were added. After 10 minutes incubation in the dark, $50 \mu$ l of solution 2 (0.1\% naphthylethylenediamine dihydrochloride) were added and incubated for 10 more minutes in the dark. The absorbance was measured at $450 \mathrm{~nm}$ using a plate reader (ELISA-Reader Infinite 200 series, Tecan, Crailsheim, Germany).

\section{Statistical analysis}

All in vitro experiments were performed in at least three independent experiments, with a minimum of three wells per experimental condition. We confirm that there is no duplication of data presentation in the text. Data are expressed as the percent of corresponding control values. The normalization to control was used because of the high variance due to the different preparations of primary cell cultures. Each data point represents mean \pm SEM. Multiple comparisons against a single reference group were performed by one-way analysis of variance (ANOVA) followed by a post hoc Dunnett's test. When all pairwise comparisons were carried out, one-way ANOVA was followed by a post hoc Student-Newman-Keuls test. For the human qPCR experiments, a Spearman correlation test between Hsp60 and TH mRNA expression was calculated. The null hypothesis was rejected at an $\alpha$ risk of $5 \%$.

\section{Results}

Hsp60 mRNA up-regulation is observed in MPTP-treated mice and in PD patients

We postulated that injury of DA neurons could result in the release of Hsp60, causing activation of microglia cells. To test this hypothesis, we first examined the dynamic changes of Hsp60 mRNA expression in the mesencephalon and the striatum of C57/Bl6 mice intoxicated with MPTP. Compared to saline-treated animals, Hsp60 mRNA expression was up-regulated in the mesencephalon and striatum of MPTP-exposed mice, the striatal response being more sustained in time (Figure 1A). Thus, this suggests that MPTP-induced mitochondrial stress in nigral 

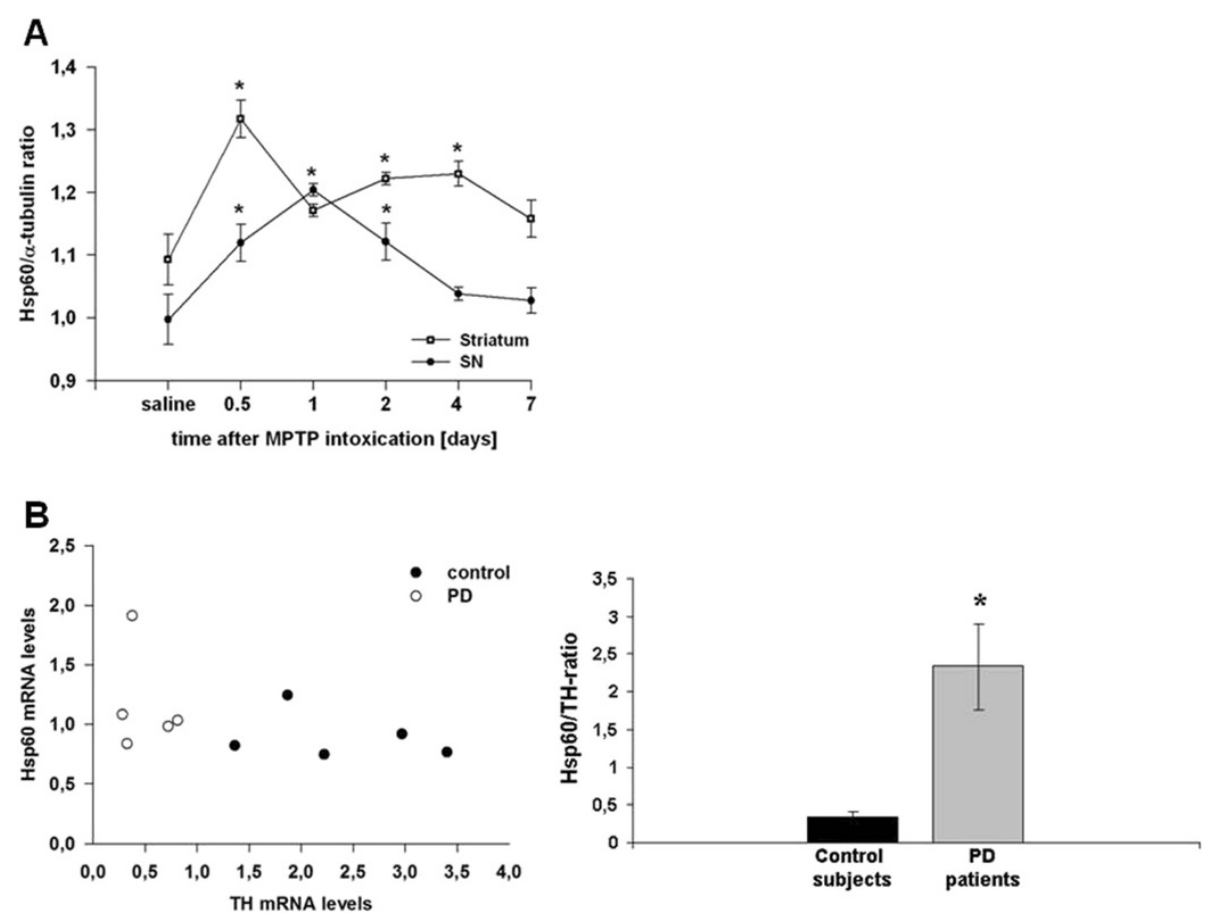

Figure 1 Hsp60 mRNA expression in 1-methyl-4-phenyl-1,2,3,6-tetrahydropyridine (MPTP)-treated mice and Parkinson disease (PD) patients. (A) Hsp60 mRNA expression in the mesencephalon (substantia nigra (SN), filled circles) and the striatum (open circles) of C57/BL6 mice treated with MPTP. Mice ( $\mathrm{n}=8$ to 10 per group) were treated using the acute MPTP paradigm $(4 \times 20 \mathrm{mg} / \mathrm{kg} \mathrm{MPTP-HCl}$ (Sigma-Aldrich, Lyon, France) every 2 hours) and animals were killed 12, 24, 48, 96 or 168 hours after the last injection. (B) Hsp60/TH mRNA ratios in the SN of PD patients and aged-matched subjects. Results were expressed as a ratio to correct for dopaminergic (DA) cell loss in PD. ${ }^{*} P<0.05$ versus corresponding controls.

DA neurons can trigger an Hsp60 response in vivo. To confront these results with the human pathology, we further assessed the expression level of Hsp60 transcripts in human post-mortem SN specimen by qRT-PCR. We found a significant increase in the Hsp60/TH ratio (to correct for DA cell loss in PD) in PD patients $(n=5)$ compared to age-matched control subjects $(n=5)$ (Figure 1B) suggesting that the Hsp60 stress response may play a role in the pathomechanism of PD.

\section{Mitochondrial stress induces up-regulation and release of Hsp60 in the human dopaminergic cell line SH-SY5Y}

To further investigate a possible link between the neuronal Hsp60 response and PD-related cellular stress, we treated the human DA cell line SH-SY5Y with the mitochondrial toxin $\mathrm{MPP}^{+}$and first assessed Hsp60 expression. As shown in Figure 2A, exposure of DA cells to $\mathrm{MPP}^{+}$ induced a time-dependent up-regulation of Hsp60 expression as assayed by immunoblot analysis on whole cell lysates. Up-regulation was first noticed 12 hours after intoxication and continuously increased up to 48 hours. The level of Hsp60 was not only increased in the intracellular compartment but also in the cell culture supernatant (Figure 2B), suggesting that cellular damage induced by mitochondrial alteration can stimulate the release of stress-related factors from diseased DA neurons. Importantly, increased extracellular Hsp60 observed at 12 and 48 hours following $\mathrm{MPP}^{+}$treatment was not due to permeabilization of cell membranes as the release of LDH (a determinant of cell membrane integrity) was only significant after 24 hours of treatment (Figure 2C). By contrast, decreased mitochondrial cell function evidenced via the MTT assay [37] was detected earlier (six hours) after $\mathrm{MPP}^{+}$treatment and concomitant with the rise in extracellular Hsp60. Altogether, these results indicate that $\mathrm{MPP}^{+}$treatment of SH-SY5Y cells compromised their function and stimulated the release of Hsp60 well before membrane rupture and death, suggesting the existence of an active Hsp60 secretory mechanism when DA neurons are in a suffering/damaged state.

\section{Hsp60-induced glial cell activation, mediates DA cell death and exacerbates MPP ${ }^{+}$toxicity in mesencephalic cultures}

We next addressed the question whether Hsp60 influences neuronal cell death and treated primary mesencephalic cultures with either $1 \mu \mathrm{M} \mathrm{MPP}{ }^{+}$at DIV 4 and DIV 5, $10 \mu \mathrm{g} / \mathrm{ml}$ hHsp60 at DIV 2 to 5 , or a combination of both. At DIV 5, medium including $\mathrm{MPP}^{+}$and hHsp60 was removed and cultures were left to recover until 


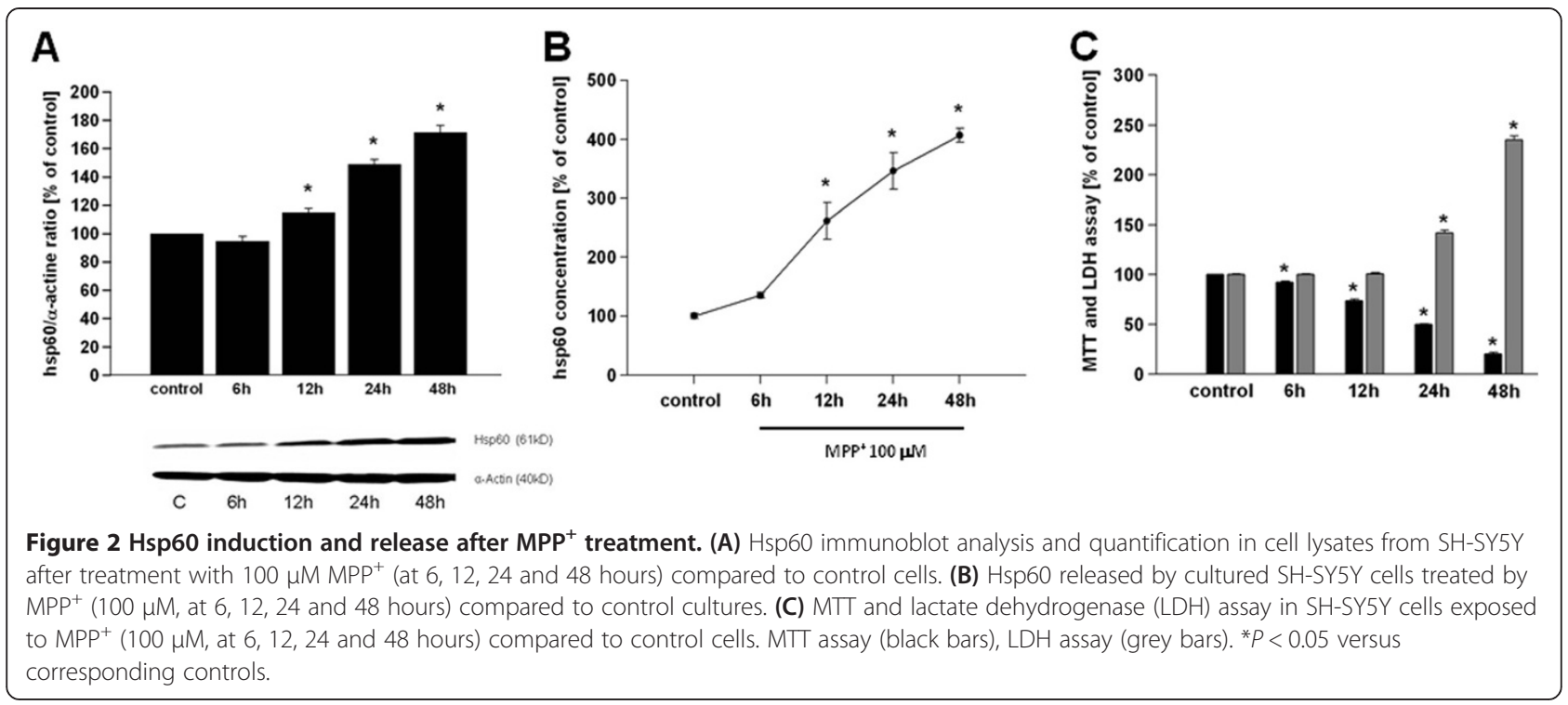

DIV 10 in control medium. Under these experimental conditions, hHsp60 treatment decreased $\mathrm{TH}^{+}$neurons to $68.3 \% \pm 2.6 \%$ compared to untreated cultures and the overall population of neurons $\left(\mathrm{NeuN}^{+}\right.$cells) of these cultures to $74.4 \% \pm 0.7 \%$ (Figure $3 \mathrm{~A}$ ). Treatment with $\mathrm{MPP}^{+}$ decreased the number of $\mathrm{TH}^{+}$cells to $50.3 \% \pm 1.4 \%$, but did not affect the number of $\mathrm{NeuN}^{+}$cells $(98.6 \% \pm 0.7 \%)$. Primary mesencephalic cultures contain only 1 to $3 \%$ of DA neurons $\left(\mathrm{TH}^{+}\right)$[38]. These neurons are selectively killed by $\mathrm{MPP}^{+}$at the dosage used, leaving intact the remaining neuronal populations. Thus, the number of $\mathrm{NeuN}^{+}$positive neurons was not significantly affected by $\mathrm{MPP}^{+}$treatment.

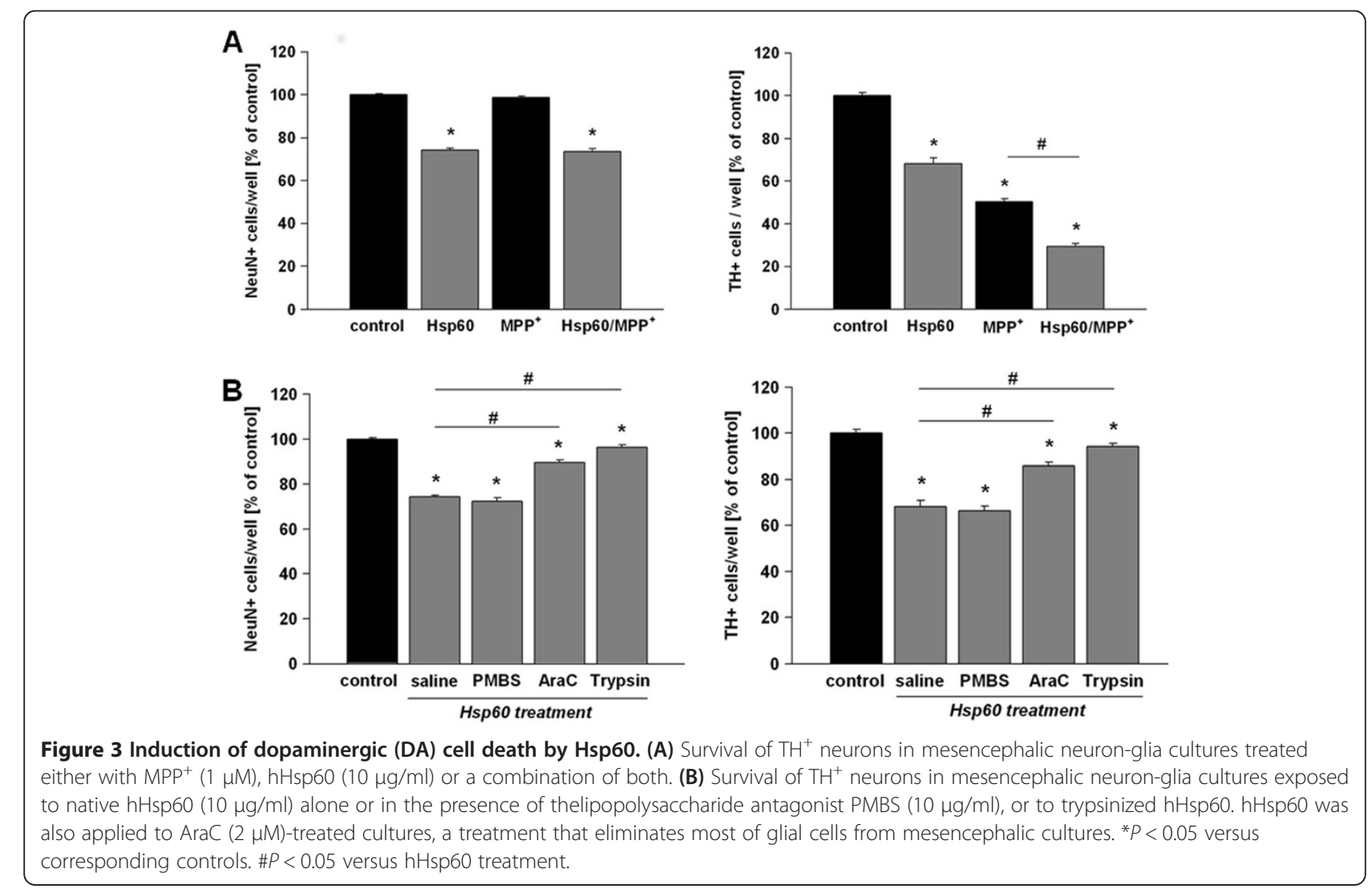


Interestingly, co-treatment with hHsp60 and $\mathrm{MPP}^{+}$led to a further loss of $\mathrm{TH}^{+}$neurons in comparison to $\mathrm{MPP}^{+}$ or hHsp60 treatment alone $\left(\mathrm{MPP}^{+}: 50.3 \% \pm 1.4 \%\right.$, hHsp60: $68.3 \% \pm 2.6$ co-treatment of $\left.\mathrm{MPP}^{+} / \mathrm{hHsp} 60: 29.4 \% \pm 1.3 \%\right)$, but exerted no additional effect on $\mathrm{NeuN}^{+}$cells (cotreatment of $\mathrm{MPP}^{+} / \mathrm{hHsp60}: 73.6 \% \pm 1.3 \%, \mathrm{MPP}^{+}: 98.6 \% \pm$ 0.7\%, hHsp60: 74.4\% \pm 0.7\%) (Figure 3A).

To determine whether neuronal cell death mediated by Hsp60 required glial cell activation, we inhibited the proliferation of glial cells with the anti-mitotic agent AraC $(2 \mu \mathrm{M})$ applied at DIV 1 for 48 hours [28]. The elimination of glial cells from the cultures curtailed neuronal cell death induced by Hsp60 (Figure 3B). Besides, hHsp60-dependent neurotoxic effects were almost abrogated when hHsp60 was previously denatured by trypsinization $\left(\mathrm{TH}^{+}: 94.3 \% \pm 1.2 \%\right)$, but not when cultures were exposed to both hHsp60 and the specific LPS antagonist PMBS $\left(\mathrm{TH}^{+}: 66.3 \% \pm 2.1 \%\right)$, suggesting that the effects of hHsp60 occurred by a mechanism unrelated to that elicited by LPS (Figure 3B).

\section{Hsp60 binds to microglia but does not induce pro-inflammatory cytokine release}

A direct biological effect of Hsp60 on microglia requires the binding to specific receptors. Therefore, to determine whether Hsp60 could provoke microglial cell-activating properties similar to those previously reported for macrophages $[39,40]$, we performed in vitro stimulation of primary microglial cell cultures with recombinant hHsp60. For comparative purposes, control cultures were exposed to LPS. Since Hsp60 is thought to induce a proinflammatory response through binding to the LPS receptor TLR4 [41], we first analyzed the binding properties of hHsp60 on murine microglia. Incubation of microglial cells with Alexa647-conjugated hHsp60, but not with Alexa647-conjugated BSA used as a negative control, revealed a punctuated distribution of bound hHsp60 suggesting the presence of cell surface receptors that binds to the protein (Figure 4A). Fluorescenceactivated cell sorting (FACS) analysis of cells incubated with increasing concentrations of hHsp60 indicated a non linear and saturable binding of hHsp60 to microglia, thus further supporting the view that microglial cells express specific Hsp60 receptors (Figure 4B).

Subsequently, microglial cells were treated with hHsp60 at a concentration of $10 \mu \mathrm{g} / \mathrm{ml}$. After 1, 2, 4, 6 and 24 hours respectively, hHsp60-exposed cells showed no morphological alterations (that is enlargement of cell bodies; data not shown). Furthermore, they did not release significantly increased amounts of pro-inflammatory cytokines (IL-6, IL-1 $\beta$ and TNF- $\alpha$ ) or NO compared to the control condition (Figure 4C). In the LPS-treated positive control $(0.1 \mu \mathrm{g} / \mathrm{ml})$, significant values of IL-6 and TNF- $\alpha$ could be shown after 6 and 24 hours respectively for NO.
However, a significant increase of IL-1 $\beta$ could not be shown in the LPS-treated group. This may be due to concentrations of LPS used being too low and/or the time of measurement being too early (six hours) to sufficiently stimulate IL- $1 \beta$ release.

In addition, microglial cells were exposed to different hHsp60 preparations (native hHsp60, hHsp60 denatured by trypsinization or boiling and hHsp60 pre-incubated with Hsp60-specific antibody or isotype control antibody). After 12 and 24 hours respectively, hHsp60-exposed cells did not release significantly increased amounts of pro-inflammatory cytokines (IL-6 and TNF- $\alpha$ ) compared to the control condition. (Figure 4D). Taken together, these observations strongly suggest that microglia express cell surface receptors to interact with Hsp60. Microglia, however, do not react to Hsp60 with the production of measurable amounts of classical pro-inflammatory cytokines such as IL-1 $\beta$, IL-6, TNF- $\alpha$ or NO release.

\section{Discussion}

Experimental data presented here suggest that Hsp60 might participate actively in DA cell demise in PD. First, in vivo, we observed that Hsp60-mRNA was up-regulated in the mouse mesencephalon and striatum in a toxinbased model of PD, as well as in the SN of PD patients. Second, in vitro, we showed that $\mathrm{MPP}^{+}$increases the expression and release of Hsp60 from DA cells and exacerbates $\mathrm{MPP}^{+}$-induced DA cell death. Surprisingly, however, despite this specific binding to microglia already been shown by Lehnardt et al. [19], hHsp60 did not cause induction of pro-inflammatory cytokines.

Hsps were initially discovered as participants in the cellular response to stress [42]. Other studies suggested that self and microbial Hsps may also play an important role in the control of the immune response [43]. In particular, Hsps including Hsp60, Hsp70 and gp96 released by injured or dying cells, are believed to function as endogenous danger signals to the immune system indicating tissue injury [44-47]. Here, we evaluated the potential role of Hsp60 in DA cell demise in PD, a pathological condition in which inflammatory processes are thought to contribute actively to degeneration.

Using the acute MPTP mouse model, characterized by a robust gliosis in the SNpc with significant up-regulation of inducible NOS and activation of microglia [48], we demonstrated that Hsp60 mRNA expression is up-regulated in the mesencephalon and the striatum after MPTP intoxication, suggesting that Hsp60 had the potential to operate as an endogenous activator of microglia in animal models of PD. Supporting this view, the kinetics of Hsp60 up-regulation after MPTP intoxication resembled that of CD11b/Mac1 induction which reflects microglial activation [48]. Interestingly, we found that in the SN of PD patients, Hsp60 mRNA also up-regulated compared to 

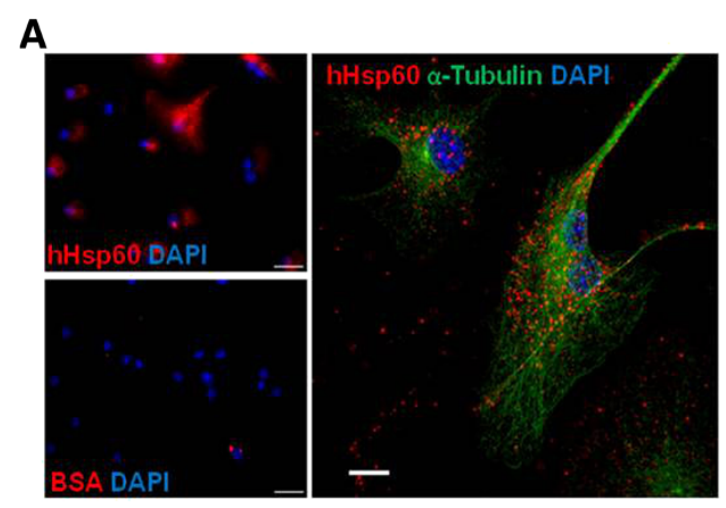

B
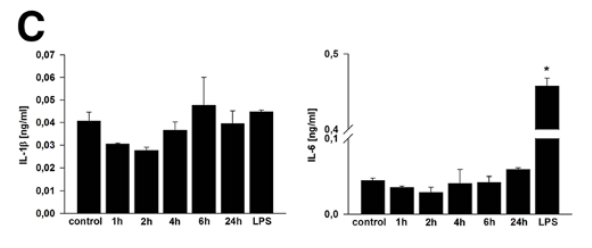

D
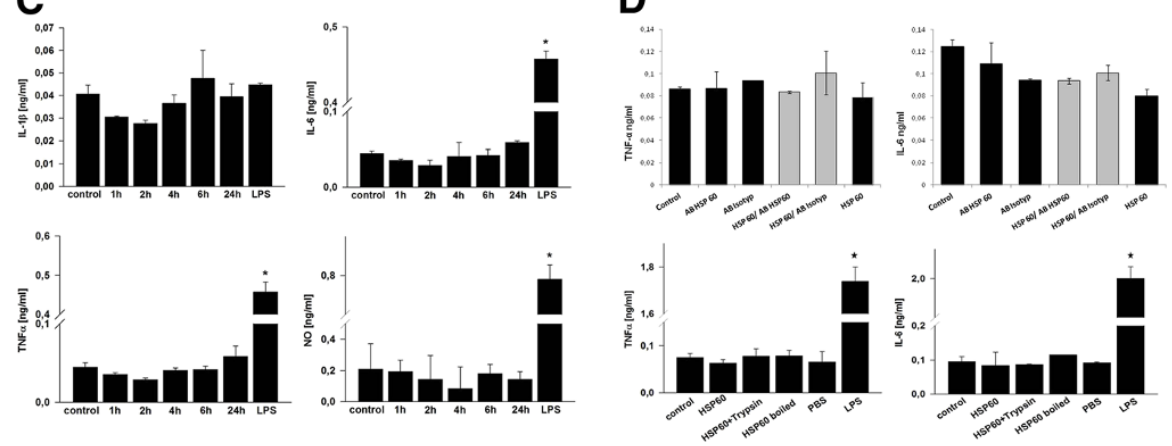

Figure 4 Hsp60 and inflammatory processes mediated by microglial cells. (A) Left: microglial cells exposed to Alexa647-conjugated hHsp60 (red label) or Alexa647-conjugated BSA (used as negative control) with 4'6-diamidino-2-phenylindole (DAPI) (blue label) counterstaining of cell nuclei. Scale bar $=50 \mu \mathrm{m}$. Right: confocal laser scanning microscopy of microglial cells that were exposed to Alexa647-conjugated Hsp60 (red label), then stained with an a-tubulin antibody (green label) and counterstained with DAPI (blue label). Scale bar = $10 \mu$ m. (B) Measure of cell surface binding of hHsp60 using extracellular FACS analysis of microglial cells labeled with an anti-Hsp60 monoclonal antibody (SPA-810,

StressGen, San Diego, CA, USA) and a phycoerythrin-labeled secondary antibody. (C) Microglial cells were treated with hHsp60 at a concentration of $10 \mu \mathrm{g} / \mathrm{ml}$, after 1, 2, 4, 6 and 24 hours respectively. Lipopolysaccharide (LPS) treatment for 6 hours for cytokine release respectively 24 hours for nitric oxide (NO)-release in a concentration of $0.1 \mu \mathrm{g} / \mathrm{ml}$ was used as positive control. (D) In addition, microglial cells were exposed to different hHsp60 preparations (native hHsp60, hHsp60 denatured by trypsinization or boiling and hHsp60 pre-incubated with Hsp60-specific antibody or isotype control antibody), after 12 and 24 hours, respectively. lipopolysaccharide (LPS) treatment for 12 hours in a concentration of $0.1 \mu \mathrm{g} / \mathrm{ml}$ was used as positive control, PBS as buffer control.

control subjects indicating that the observations made with the MPTP mouse model might be of relevance for the human pathology.

The assumption that Hsp60 could intervene actively in the progression of the disease was also supported by several observations made in two complementary cell culture settings; (i) we found that mitochondrial stress triggered by $\mathrm{MPP}^{+}$caused an up-regulation and release of Hsp60 in human DA SH-SY5Y cells well before the onset of neuronal cell demise; in our model system, the release of Hsp60 was already detectable in the supernatant of cells with intact plasma membranes, so that cell lysis would not be necessary for the accumulation of Hsp60 in the extracellular milieu, (ii) application of Hsp60 to primary mesencephalic cultures triggered DA cell death per se and importantly, it also exacerbated the toxicity of $\mathrm{MPP}^{+}$for these neurons.
The stimulation of TLR4 by LPS induces the release of critical pro-inflammatory cytokines that are necessary to activate potent immune responses. Recently, we could demonstrate that TLR4-deficient mice are less vulnerable to MPTP intoxication than wild-type mice and display a decreased number of activated microglial cells after MPTP application, suggesting that the TLR4 pathway is involved in experimental PD. However, the endogenous activator of TLR4 in this scenario could not be identified [49]. A number of arguments suggested that Hsp60 could be a potential ligand for TLR4, which is expressed on microglia, astrocytes and endothelial cells in the central nervous system [50-53]. In particular, extracellular eukaryotic Hsp60 was found to induce the release of pro-inflammatory cytokines such as IL-1, IL-6, IL-12, and TNF- $\alpha$ in dendritic cells and macrophages $[43,45,54,55]$. Lehnardt et al. determined in a previous study that the neurotoxic effect of 
Hsp60 requires the presence of microglia. Additionally, Lehnardt et al. could show that microglia bind Hsp60Alexa, whereas neurons, oligodendrocytes and astrocytes did not show any labeling with Hsp60-Alexa [19]. Therefore, we have placed the focus in our study on microglia and not on astrocytes or endothelial cells.

Here, we observed an induction of Hsp60 after MPTP intoxication. Furthermore, we established that Hsp60 binds specifically to microglia in a saturable-dependent manner, suggesting that the protein could operate as an endogenous activator of TLR4, thus stimulating inflammatory processes. In contrast to the latter, Hsp60 did not induce the release of detectable amounts of cytokines. However, low levels of pro-inflammatory cytokines that are below detection limit may be induced by Hsp60 and might well exert biological activity in a certain microenvironment: that is the direct contact of microglia with neighbouring neuronal cells. In addition, Hsp60 may induce the production of stimulatory or toxic molecules other than IL-1 $\beta$, IL-6, TNF- $\alpha$ or NO that were not analyzed in this study.

Taking into consideration that Hsp60 activates murine antigen-presenting cells (APC) also via TLR4 independent pathways in the absence of LPS or other so called pathogen-associated molecular patterns (PAMPs) [41,56], it is also possible that the Hsp60 acts via other receptors such as the lectin-like oxidized low-density lipoprotein receptor-1 (LOX-1) previously identified as a putative receptor for Hsp60 in microglial cells [57]. Other PAMP could be involved in the stimulation of the innate immune system in some types of sterile inflammation, like PD, which need to be explored in further studies.

Alternatively, a direct effect of Hsp60 on DA neurons remains also conceivable. Yet, the requirement of glial cells in the effects of Hsp60 appeared crucial as its toxic effects towards DA neurons were curtailed in neuronalenriched cultures.

\section{Conclusions}

Taken together, we could demonstrate a deleterious role of Hsp60 on DA neurons in different experimental PD models. However, our data do not support that Hsp60 is an endogenous activator of the TLR4-pathway in experimental PD models and, by extrapolation, in the human disease. Thus the cellular target of Hsp60 remains to be identified.

\footnotetext{
Abbreviations

ANOVA: analysis of variance; APC: antigen-presenting cells; Ara-C: cytosine arabinoside; BSA: bovine serum albumin; CD11b: cluster of differentiation molecule 11B; CNS: central nervous system; DA: dopaminergic; DAPI: 4'6diamidino-2-phenylindole; DIV: days in vitro; DMEM: Dulbecco's Modified Eagle's Medium; DNA: deoxyribonucleic acid; ECM: major extracellular matrix; ELISA: Enzyme Linked Immunosorbent Assay; FACS: Fluorescence-activated cell sorting; FCS: fetal calf serum; GAPDH: Glyceraldehyde 3-phosphate dehydrogenase; hHsp60: human heat shock protein 60; HPRT: Hypoxanthineguanine phosphoribosyltransferase; HRP: Horseradish peroxidase; Hsp60: heat shock protein 60; Hsps: heat shock proteins; IL-1ß: interleukin-1 beta;
}

IL-6: interleukin-6; LDH: lactate dehydrogenase; LPS: lipopolysaccharide; Mac1: Macrophage-1 antigen; MK-801: Dizocilpine; MPP : 1-methyl-4phenylpyridinium; MPTP: 1-methyl-4-phenyl-1,2,3,6-tetrahydropyridine; MTT: 3-[4,5-dimethylthiazol-2-yl]-2,5-diphenyl-tetrazolium bromide; MyD88: myeloid differentiation factor 88; NeuN: neuronal Nuclei; NO: nitric oxide; PAMPs: pathogen-associated molecular patterns; PBS: phosphate buffered saline; PD: Parkinson disease; PEl: polyethylenimine; PFA: paraformaldehyde; PMBS: polymyxin B; RNA: ribonucleic acid; SH-SY5Y: human neuroblastoma cell line; SN: substantia nigra; SNpc: substantia nigra pars compacta; TH: tyrosine hydroxylase; TLR4: Toll-like receptor 4; TLRs: Toll-like receptors; TNF-a: tumor necrosis factor-alpha.

\section{Competing interests}

The authors declare that they have no competing interests.

\section{Authors' contributions}

The work presented here was carried out in collaboration between all authors. $\mathrm{CN}, \mathrm{SH}$ and $\mathrm{AH}$ defined the research theme. $\mathrm{CN}, \mathrm{SH}$ and $\mathrm{AH}$ designed methods and experiments, carried out the laboratory experiments, analyzed the data, interpreted the results and wrote the paper. $L M, D A F, T L$, $\mathrm{CH}$ and $\mathrm{LL}$ co-designed experiments, discussed analyses, interpretation, and presentation. RCD and ECH analyzed the data and interpreted the results. AO, $\mathrm{MB}$ and PPM co-worked on associated data collection and their interpretation. All authors have contributed, seen and approved the manuscript.

\section{Acknowledgements}

CN was supported by a postdoctoral grant from the Deutsche Forschungsgemeinschaft, (DFG), Germany. DAF was supported by a grant from the Michael J Fox Foundation (MJFF) and from the University Medical Center Giessen and Marburg (UKGM). AO was supported by the MildredScheel-Stiftung. TL was supported by a Master grant from the Fondation pour la Recherche Médicale (FRM). CH was supported by an MD thesis grant by the Boehringer Ingelheim Fond (BIF). PPM is supported by program 'Investissements d'avenir' ANR-10-IAIHU-06. ECH and SH are investigators at the Centre National pour la Recherche Scientifique (CNRS). AH was supported by a 'Poste Vert' (Accueil de Chercheurs Etrangers) from the Institut National de la Santé et de la Recherche Médicale (INSERM). The research leading to these results has received funding from the program 'Investissements d'avenir' ANR-10-IAIHU-06.

\section{Author details}

${ }^{1}$ CR-ICM, INSERM UMR_S1127, Université Pierre et Marie Curie Paris 06 UMR_S1127, CNRS UMR 7225, Groupe Hospitalier Pitié-Salpêtrière, 75013 Paris, France. ${ }^{2}$ Department of Neurology, Philipps-University Marburg, 35043 Marburg, Germany. ${ }^{3}$ Bernhard Nocht Institute for Tropical Medicine, Hamburg 20324, Germany. ${ }^{4}$ Institute of Neurogenetics, University of Lübeck, 23562 Lübeck, Germany. ${ }^{5}$ Department of Psychiatry, University of Lübeck, 23538 Lübeck, Germany.

Received: 20 November 2013 Accepted: 29 April 2014

Published: 8 May 2014

\section{References}

1. Fahn SPS: Parkinsonism. In Meritt's Neurology. 10th edition. Edited by Rowland LP. New York: Lippincott; 2000:679-693.

2. Hirsch EC, Hunot S: Neuroinflammation in Parkinson's disease: a target for neuroprotection? Lancet Neurol 2009, 8:382-397.

3. McGeer PL, Itagaki S, Boyes BE, McGeer EG: Reactive microglia are positive for HLA-DR in the substantia nigra of Parkinson's and Alzheimer's disease brains. Neurology 1988, 38:1285-1291

4. Barcia C, Sanchez Bahillo A, Fernandez-Villalba E, Bautista V, Poza YPM, Fernandez-Barreiro A, Hirsch EC, Herrero MT: Evidence of active microglia in substantia nigra pars compacta of Parkinsonian monkeys 1 year after MPTP exposure. Glia 2004, 46:402-409.

5. Langston JW, Forno LS, Tetrud J, Reeves AG, Kaplan JA, Karluk D: Evidence of active nerve cell degeneration in the substantia nigra of humans years after 1-methyl-4-phenyl-1,2,3,6-tetrahydropyridine exposure. Ann Neurol 1999, 46:598-605.

6. McGeer PL, Schwab C, Parent A, Doudet D: Presence of reactive microglia in monkey substantia nigra years after 1-methyl-4-phenyl-1,2,3,6tetrahydropyridine administration. Ann Neurol 2003, 54:599-604. 
7. Laflamme N, Rivest S: Toll-like receptor 4: the missing link of the cerebral innate immune response triggered by circulating gram-negative bacterial cell wall components. Faseb J 2001, 15:155-163.

8. Kawai T, Akira S: Pathogen recognition with Toll-like receptors. Curr Opin Immunol 2005, 17:338-344

9. Tsan MF, Gao B: Endogenous ligands of Toll-like receptors. J Leukoc Biol 2004, 76:514-519.

10. Erridge C: Endogenous ligands of TLR2 and TLR4: agonists or assistants? J Leukoc Biol 2010, 87:989-999.

11. Sharp FR, Massa SM, Swanson RA: Heat-shock protein protection. Trends Neurosci 1999, 22:97-99.

12. Barreto A, Gonzalez JM, Kabingu E, Asea A, Fiorentino S: Stress-induced release of HSC70 from human tumors. Cell Immunol 2003, 222:97-104.

13. Lang A, Benke D, Eitner F, Engel D, Ehrlich S, Breloer M, Hamilton-Williams E, Specht S, Hoerauf A, Floege J, von Bonin A, Kurts C: Heat shock protein 60 is released in immune-mediated glomerulonephritis and aggravates disease: in vivo evidence for an immunologic danger signal. J Am Soc Nephrol 2005, 16:383-391.

14. Rajaiah R, Moudgil KD: Heat-shock proteins can promote as well as regulate autoimmunity. Autoimmun Rev 2009, 8:388-393.

15. Schapira AH: Mitochondria in the aetiology and pathogenesis of Parkinson's disease. Lancet Neurol 2008, 7:97-109.

16. Pfister $G$, Stroh CM, Perschinka H, Kind M, Knoflach M, Hinterdorfer P, Wick $\mathrm{G}$ : Detection of HSP60 on the membrane surface of stressed human endothelial cells by atomic force and confocal microscopy. J Cell Sci 2005, 118:1587-1594.

17. Sapozhnikov AM, Ponomarev ED, Tarasenko TN, Telford WG: Spontaneous apoptosis and expression of cell surface heat-shock proteins in cultured EL-4 lymphoma cells. Cell Prolif 1999, 32:363-378.

18. Ohashi K, Burkart V, Flohe S, Kolb H: Cutting edge: heat shock protein 60 is a putative endogenous ligand of the toll-like receptor-4 complex. J Immunol 2000, 164:558-561.

19. Lehnardt S, Schott E, Trimbuch T, Laubisch D, Krueger C, Wulczyn G, Nitsch $R$, Weber JR: A vicious cycle involving release of heat shock protein 60 from injured cells and activation of toll-like receptor 4 mediates neurodegeneration in the CNS. J Neurosci 2008, 28:2320-2331.

20. Jackson-Lewis V, Przedborski S: Protocol for the MPTP mouse model of Parkinson's disease. Nat Protoc 2007, 2:141-151.

21. Sonsalla PK, Heikkila RE: The influence of dose and dosing interval on MPTP-induced dopaminergic neurotoxicity in mice. Eur J Pharmacol 1986, 129:339-345.

22. Tanaka T, lqbal K, Trenkner E, Liu DJ, Grundke-labal I: Abnormally phosphorylated tau in SY5Y human neuroblastoma cells. FEBS Lett 1995, 360:5-9.

23. Gomez C, Reiriz J, Pique M, Gil J, Ferrer I, Ambrosio S: Low concentrations of 1-methyl-4-phenylpyridinium ion induce caspase-mediated apoptosis in human SH-SY5Y neuroblastoma cells. J Neurosci Res 2001, 63:421-428.

24. Michel PP, Agid Y: Chronic activation of the cyclic AMP signaling pathway promotes development and long-term survival of mesencephalic dopaminergic neurons. J Neurochem 1996, 67:1633-1642.

25. Salthun-Lassalle B, Hirsch EC, Wolfart J, Ruberg M, Michel PP: Rescue of mesencephalic dopaminergic neurons in culture by low-level stimulation of voltage-gated sodium channels. J Neurosci 2004 24:5922-5930.

26. Knusel B, Michel PP, Schwaber JS, Hefti F: Selective and nonselective stimulation of central cholinergic and dopaminergic development in vitro by nerve growth factor, basic fibroblast growth factor, epidermal growth factor, insulin and the insulin-like growth factors I and II. J Neurosci 1990, 10:558-570.

27. Kawamoto JC, Barrett JN: Cryopreservation of primary neurons for tissue culture. Brain Res 1986, 384:84-93.

28. Mourlevat S, Troadec JD, Ruberg M, Michel PP: Prevention of dopaminergic neuronal death by cyclic AMP in mixed neuronal/glial mesencephalic cultures requires the repression of presumptive astrocytes. Mol Pharmacol 2003, 64:578-586.

29. Michel PP, Dandapani BK, Knusel B, Sanchez-Ramos J, Hefti F: Toxicity of 1-methyl-4-phenylpyridinium for rat dopaminergic neurons in culture: selectivity and irreversibility. J Neurochem 1990, 54:1102-1109.

30. Saura J, Tusell JM, Serratosa J: High-yield isolation of murine microglia by mild trypsinization. Glia 2003, 44:183-189.
31. Mattson MP, Barger SW, Begley JG, Mark RJ: Calcium, free radicals, and excitotoxic neuronal death in primary cell culture. Methods Cell Biol 1995 46:187-216

32. Van Meerloo J, Kaspers GJ, Cloos J: Cell sensitivity assays: the MTT assay. Methods Mol Biol 2011, 731:237-245

33. Stockert JC, Blazquez-Castro A, Canete M, Horobin RW, Villanueva A: MTT assay for cell viability: intracellular localization of the formazan product is in lipid droplets. Acta Histochem 2012, 114:785-796.

34. Medzhitov R: Toll-like receptors and innate immunity. Nat Rev Immunol 2001, 1:135-145.

35. Poltorak A, He X, Smirnova I, Liu MY, Van Huffel C, Du X, Birdwell D, Alejos E, Silva M, Galanos C, Freudenberg M, Ricciardi-Castagnoli P, Layton B, Beutler $\mathrm{B}$ : Defective LPS signaling in $\mathrm{C} 3 \mathrm{H} / \mathrm{HeJ}$ and $\mathrm{C} 57 \mathrm{BL} / 10 \mathrm{ScCr}$ mice: mutations in Tlr4 gene. Science 1998, 282:2085-2088.

36. Amano F, Noda T: Improved detection of nitric oxide radical (NO.) production in an activated macrophage culture with a radical scavenger, carboxy PTIO and Griess reagent. FEBS Lett 1995, 368:425-428.

37. Jang YO, Quan X, Das R, Xu S, Chung CH, Ahn CM, Baik SK, Kong ID, Park KS, Kim MY: High-dose clevudine impairs mitochondrial function and glucose-stimulated insulin secretion in INS-1E cells. BMC Gastroenterol 2012, 12:4

38. Michel PP, Ruberg M, Agid Y: Rescue of mesencephalic dopamine neurons by anticancer drug cytosine arabinoside. J Neurochem 1997 69:1499-1507

39. Habich C, Baumgart K, Kolb H, Burkart V: The receptor for heat shock protein 60 on macrophages is saturable, specific, and distinct from receptors for other heat shock proteins. J Immunol 2002, 168:569-576.

40. Vabulas RM, Ahmad-Nejad P, da Costa C, Miethke T, Kirschning CJ, Hacker H, Wagner $\mathrm{H}$ : Endocytosed HSP60s use toll-like receptor 2 (TLR2) and TLR4 to activate the toll/interleukin-1 receptor signaling pathway in innate immune cells. J Biol Chem 2001, 276:31332-31339.

41. Osterloh A, Kalinke U, Weiss S, Fleischer B, Breloer M: Synergistic and differential modulation of immune responses by $\mathrm{Hsp} 60$ and lipopolysaccharide. J Biol Chem 2007, 282:4669-4680.

42. Welch WJ: Heat shock proteins functioning as molecular chaperones: their roles in normal and stressed cells. Philos Trans R Soc Lond B Biol Sci 1993, 339:327-333.

43. Quintana FJ, Cohen IR: The HSP60 immune system network. Trends Immunol 2011, 32:89-95.

44. Breloer M, Dorner B, More SH, Roderian T, Fleischer B, von Bonin A: Heat shock proteins as 'danger signals': eukaryotic Hsp60 enhances and accelerates antigen-specific IFN-gamma production in T cells. Eur J Immunol 2001, 31:2051-2059.

45. Chen W, Syldath U, Bellmann K, Burkart V, Kolb H: Human 60-kDa heat-shock protein: a danger signal to the innate immune system. J Immunol 1999, 162:3212-3219.

46. Gallucci S, Matzinger P: Danger signals: SOS to the immune system. Curr Opin Immunol 2001, 13:114-119.

47. Wallin RP, Lundqvist A, More SH, von Bonin A, Kiessling R, Ljunggren HG Heat-shock proteins as activators of the innate immune system. Trends Immunol 2002, 23:130-135.

48. Liberatore GT, Jackson-Lewis V, Vukosavic S, Mandir AS, Vila M, McAuliffe WG, Dawson VL, Dawson TM, Przedborski S: Inducible nitric oxide synthase stimulates dopaminergic neurodegeneration in the MPTP model of Parkinson disease. Nat Med 1999, 5:1403-1409.

49. Noelker C, Morel L, Lescot T, Osterloh A, Alvarez-Fischer D, Breloer M, Henze C, Depboylu C, Skrzydelski D, Michel PP, Dodel RC, Lu L, Hirsch EC, Hunot S, Hartmann A: Toll like receptor 4 mediates cell death in a mouse MPTP model of Parkinson disease. Sci Rep 2013, 3:1393.

50. Fellner L, Irschick R, Schanda K, Reindl M, Klimaschewski L, Poewe W, Wenning GK, Stefanova N: Toll-like receptor 4 is required for alpha-synuclein dependent activation of microglia and astroglia. Glia 2013, 61:349-360.

51. Jayakumar AR, Tong XY, Curtis KM, Ruiz-Cordero R, Abreu MT, Norenberg MD: Increased toll-like receptor 4 in cerebral endothelial cells contributes to the astrocyte swelling and brain edema in acute hepatic encephalopathy. J Neurochem 2014, 128:890-903.

52. Pascual-Lucas M, Fernandez-Lizarbe S, Montesinos J, Guerri C: LPS or ethanol triggers clathrin- and rafts/caveolae-dependent endocytosis of TLR4 in cortical astrocytes. J Neurochem 2014, 129:448-462.

53. Tarassishin L, Suh HS, Lee SC: LPS and IL-1 differentially activate mouse and human astrocytes: role of CD14. Glia 2014, 62:999-1013. 
54. Flohe SB, Bruggemann J, Lendemans S, Nikulina M, Meierhoff G, Flohe S, Kolb H: Human heat shock protein 60 induces maturation of dendritic cells versus a Th1-promoting phenotype. J Immunol 2003, 170:2340-2348.

55. Kol A, Lichtman AH, Finberg RW, Libby P, Kurt-Jones EA: Cutting edge: heat shock protein (HSP) 60 activates the innate immune response: CD14 is an essential receptor for HSP60 activation of mononuclear cells. J Immunol 2000, 164:13-17.

56. Osterloh A, Veit A, Gessner A, Fleischer B, Breloer M: Hsp60-mediated T cell stimulation is independent of TLR4 and IL-12. Int Immunol 2008, 20:433-443.

57. Zhang D, Sun L, Zhu H, Wang L, Wu W, Xie J, Gu J: Microglial LOX-1 reacts with extracellular HSP60 to bridge neuroinflammation and neurotoxicity. Neurochem Int 2012, 61:1021-1035.

doi:10.1186/1742-2094-11-86

Cite this article as: Noelker et al: Heat shock protein 60: an endogenous inducer of dopaminergic cell death in Parkinson disease. Journal of

Neuroinflammation 2014 11:86.

\section{Submit your next manuscript to BioMed Central and take full advantage of:}

- Convenient online submission

- Thorough peer review

- No space constraints or color figure charges

- Immediate publication on acceptance

- Inclusion in PubMed, CAS, Scopus and Google Scholar

- Research which is freely available for redistribution 